\title{
ANÁLISE DA NECESSIDADE DE DRENAGEM ABDOMINAL E COMPLICAÇÕES PÓS CIRÚRGICAS DE APENDICECTOMIA EM RELAÇÃO AO TEMPO DE EVOLUÇÃ̃O ENTRE OS ANOS DE 2016 E 2017 NO HOSPITAL REGIONAL DO GAMA - DF (HRG-DF).
}

\author{
ANALYSIS OF THE NEED FOR ABDOMINAL DRAINAGE AND POST SURGICAL COMPLICATIONS OF APENDICECTOMY \\ IN RELATION TO THE TIME OF EVOLUTION BETWEEN THE YEARS OF 2016 AND 2017 NON HOSPITAL REGIONAL DO \\ GAMA - DF (HRG-DF).
}

Guilherme Augusto Olly de Souza Costa, José Carlos Delfino, Thaynara Giovanna Tito Delfino, Mario Sales Neves do Carmo Filho, Natascha Mourão Moreira

DOI - 10.5935/2236-5117.2021v58a33

\section{RESUMO}

Introdução: A apendicite é a causa mais comum de dor abdominal aguda, que requer intervenção cirúrgica, sendo mais comum em adultos jovens. Seu diagnóstico precoce é essencial para reduzir a morbidade. Sua abordagem cirúrgica vai progressivamente tornando-se mais complexa, sobretudo após as primeiras 48 horas.

Objetivo: Comparar a evolução no pós-operatório de pacientes com dreno abdominal na apendicite complicada, com aqueles que não tiveram a cavidade abdominal drenada.

Método: Trata-se de um estudo transversal retrospectivo dos pacientes submetidos a apendicectomia de janeiro de 2016 a dezembro de 2017 a partir do livro de registro de cirurgias de urgências do HRG, banco de dados do sistema eletrônico Track Care.

Resultados: Entre os anos de 2016 e 2017 foram realizadas 374 cirurgias de apendicectomia pela emergência no Hospital Regional do Gama (HRG). Em 2016, 184 realizadas e em 2017, 190, um acréscimo de 3,2\% em relação ao ano anterior. A doença foi mais prevalente em faixa etária menor ou igual a 30 anos, cerca de $58,8 \%$, é mais frequente em homens jovens com $68,7 \%$ dos casos analisados. Em torno de $48 \%$ dos pacientes submetidos a apendicectomia tiveram complicações.

Conclusão: No presente estudo foi observado que a maioria da população acometida pela patologia são de adultos jovens do sexo masculino. Indivíduos com idade mais avançada tem maior tempo de evolução clínica e consequentemente maiores taxas de apendicite complicada. $O$ uso do dreno abdominal na apendicite não apresentou muitos benefícios e acarretou ao paciente maior taxa de complicações pós-operatórias.

Palavras-chave: Drenagem. Abscesso Abdominal. Apendicite. Infecção da Ferida Cirúrgica
Guilherme Augusto Olly de Souza Costa -UNICEPLAC, Medicina - Gama - DF - Brasil

José Carlos Delfino - UNICEPLAC, Medicina - Gama - DF - Brasil

Thaynara Giovanna Tito Delfino - UNICEPLAC, Medicina - Gama - DF - Brasil

Mario Sales Neves do Carmo Filho - Hospital Regional do Gama, Cirurgia Greal - Gama - DF - Brasil

Natascha Mourão Moreira - Hospital Regional do Gama, Cirurgia Greal - Gama - DF - Brasil

Correspondência: Quadra 55 Lote 15/17 Ed. Tamisa "E" apartamento, 322 - Setor Central - Gama, DF. Cep: 72.405-560 Telefone: (61) 3257-5026 / (38)99169-7685

Internet: guilherme_olly@hotmail.com

Conflito de interesses: não existem conflitos de interesse.

\section{ABSTRACT}

Introduction: Appendicitis is the most common cause of acute abdominal pain, which requires surgical intervention, being more common in young adults. Early diagnosis is essential to reduce morbidity. His surgical approach is progressively becoming more complex, especially after the first 48 hours.

Objective: To compare the postoperative evolution of patients with an abdominal drain in complicated appendicitis, with those who did not have their abdominal cavity drained.

Method: This is a retrospective cross-sectional study of patients who underwent appendectomy from January 2016 to December 2017 from the HRG emergency surgery record book, the electronic database of the Track Care electronic system.

Results: Between the years 2016 and 2017, 374 appendectomy surgeries were performed for the emergency 
at Hospital Regional do Gama (HRG). In 2016, 184 performed and in 2017,190 , an increase of $3.2 \%$ in relation to the previous year. The disease was more prevalent in the age group less than or equal to 30 years, about $58.8 \%$, it is more frequent in young men with $68.7 \%$ of the cases analyzed. Around $48 \%$ of patients undergoing appendectomy had complications.

Conclusion: In the present study, it was observed that the majority of the population affected by the pathology are young adult males. Individuals with older age have longer clinical evolution and consequently higher rates of complicated appendicitis. The use of the abdominal drain in appendicitis did not present many benefits and resulted in a higher rate of postoperative complications for the patient.

Keywords: Flood-Bypass Channel. Abdominal Abscess. Appendicitis

\section{INTRODUÇÃO}

A apendicite aguda é a principal cirurgia abdominal não eletiva realizada, sendo umas das patologias abdominais mais frequentes, apresentando uma incidência de 48,1 por dez mil habitantes, por ano. ${ }^{1,2}$ Sua morbidade chega a $10 \%$ e mortalidade que varia de $0,24 \%$ a $4 \%$. Sua incidência ocorre entre as idades de 10 e 30 anos, com pico de 10-14 anos no sexo feminino e 15-19 anos no sexo masculino, em uma razão de $3: 2$ respectivamente. ${ }^{3,4,5}$

As causas de apendicite aguda, apesar de serem desconhecidas, acredita-se que a obstrução do lúmen por fezes espessadas, hiperplasia linfoide, matéria vegetal ou sementes, parasitas ou neoplasma, seja a maior sua maior etiologia. A obstrução provoca acúmulo de secreção continuada de muco, levando a proliferação bacteriana local, distensão intraluminal e isquemia por má perfusão vascular. 0 processo inflamatório progride, propiciando a formação gangrena, perfuração, abscesso intraperitoneais, peritonite, choque e até mesmo o óbito. ${ }^{6}$

Como sintoma mais precoce e comum, é relatado o desconforto periumbilical e mesogástrio, devido ativação o sistema nervoso aferente do peritônio visceral do apêndice vermiforme. Com a progressão do quadro, ocorre uma inflamação do peritônio parietal sobrejacente do apêndice, tornando a dor localizada em fossa ilíaca direita abdominal. Outros sintomas associados bastante frequentes são: vômitos, febre e hiporexia. Sintomas menos frequentes também podem estar presentes como hematúria microscópica, diarreia ou constipação intestinal. ${ }^{6,7}$
Por vezes, exames laboratoriais são necessários para complementação diagnóstica e exclusão de diagnósticos diferenciais. Leucocitose com desvio a esquerda pode estar presente com mais de $75 \%$ dos casos de apendicite, completando assim os critérios clínicos do escore de Alvarado. Este escore foi estabelecido como diretriz que unem aspectos clínicos e laboratoriais para diagnóstico e manejo da propedêutica dos pacientes com possível quadro de apendicite aguda. ${ }^{8} \mathrm{Em}$ persistência da dúvida diagnóstica,pode-seabrirmãodeexamesdeimagemcomo radiografia simples de abdome agudo, ultrassonografia abdominal e a tomografia computadorizada de abdome, sendo esta considerada melhor escolha de imagem, com sensibilidade de $80 \%$ e especificidade de $80-90 \% .7,9,10$

Como conduta sobre a suspeita diagnóstica de apendicite aguda, a apendicectomia é o tratamento de escolha, seja por técnica aberta ou via laparoscópica. No intraoperatório, já é possível caracterizar o aspecto macroscópico do apêndice cecal e dessa forma categorizar a apendicite aguda em não complicada ou complicada, sendo as elas: necrose, perfuração, abscesso localizado e peritonite franca. ${ }^{3,7} \mathrm{O}$ diagnóstico e tratamento precoce reduzem o tempo de internação hospitalar e o risco de complicações pós-operatórias, tais como infecção de ferida operatória, abscesso localizado e deiscência de aponeurose. ${ }^{11,12,13}$

Nas apendicites complicadas, o uso de drenos abdominais costuma ser uma prática frequente. 0 sítio com o dreno na cavidade abdominal após a apendicectomia é um fator de risco para o surgimento de infecções pósoperatórias, que é a complicação mais frequente. ${ }^{14}$

O uso do dreno abdominal é feito na tentativa de monitorar, resolver ou até mesmo prevenir a formação de abscesso intracavitário. 0 seu uso racionalizado vem acontecendo nas últimas décadas e hoje é discutido o tipo de dreno, a funcionalidade e o momento mais adequado para usá-lo. 0 seu mecanismo depende da intenção de uso, tempo, local da introdução e do tipo de cirurgia a qual o paciente será submetido. ${ }^{14,15}$

O dreno abdominal possui indicadores que facilitam a sua prescrição ou sua contraindicação. Recomenda-se seu uso para drenagem de secreções delimitadas como coleções serosas ou abscessos, levando em consideração a necessidade para conter um único foco infeccioso. $\mathrm{Na}$ presença de peritonite difusa, por exemplo, a presença do dreno está contraindicada. ${ }^{14,15}$

A implantação do dreno nos sítios específicos pode acarretar complicações imanente a essa condição. 
Além disso, a infecção de sítio cirúrgico ainda tem valor preditivo para avaliar o tempo de internação do paciente e os custos subsequentes, já que são propostos novos planos de cuidados para os pacientes. ${ }^{15}$

Com o índice de mortalidade pós-cirúrgica diminuindo e com a qualidade de vida aumentando, viu-se a necessidade da introdução de novos parâmetros que pudessem avaliar a qualidade e a evolução pósoperatória dos pacientes. Neste contexto, Clavien et al., em 1992, surgiu com uma classificação de complicações cirúrgicas que a princípio foi idealizada para colecistectomia. A base para formular essa classificação foi o tratamento proposto para o pós- operatório e a gravidade das suas complicações. Em 2004, Dindo et al. trouxe reformulações a classificação idealizada pelo grupo de Clavien.

A classificação se divide em 5 graus, os quais são, respectivamente: I- Qualquer desvio do curso pósoperatório Ideal sem necessidade de tratamento farmacológico ou de intervenções cirúrgicas, endoscópicas e radiológicas; II- Requer tratamento farmacológico com drogas diferentes daquelas permitidas para complicações grau l; III-Exige intervenção cirúrgica, endoscópica ou intervenção radiológica; IV- Complicação com Risco de vida (incluindo SNC) * Necessidade de UTI; V- Morte do Paciente. ${ }^{16}$

Por fim, o presente estudo tem por objetivo comparar a evolução do pós-operatório de pacientes com dreno abdominal na apendicite aguda complicada, com aqueles que não tiveram a cavidade abdominal drenada, no serviço de Cirurgia Geral do Hospital Regional do Gama - Distrito Federal, no período de janeiro de 2016 a dezembro de 2017.

\section{HIPÓTESE CIENTIIFICA}

A utilização dos drenos cirúrgicos abdominais quando solicitado já nos remete a presença de alguma coleção indesejada, como sangue, abscesso, bile e substâncias que possam extravasar no pós-cirúrgico. Com isso, o paciente fica sujeito a possíveis infecções e necroses

teciduais. Entretanto, o dreno pode ter uma função profilática que é a detecção precoce de hemorragias e fístulas.

Tendo como objetivo principal do estudo a comparação das complicações dos pacientes com e sem dreno abdominal. Observamos que o tempo de internação, o número e o tipo de complicações nos pacientes com dreno é mais frequente em relação ao paciente sem o dreno. Diener et al., por meio de meta-análise, observaram que as complicações oriundas de drenagens estão relacionadas, principalmente, com o tempo de permanência do dreno na cavidade.

Em análise secundária podemos ainda perceber que o número de intervenções cirúrgicas está intimamente relacionado.

\section{OBJETIVOS}

\section{Geral}

Comparar a evolução no pós-operatório de pacientes com dreno abdominal na apendicite complicada, com aqueles que não tiveram a cavidade abdominal drenada.

\section{Específicos}

Comparar os dois grupos quanto aos: dias de internação hospitalar, uso de antibioticoterapia, complicações pósoperatórias, tipos de complicações pós-operatórias e necessidade de reabordagem cirúrgica;

Avaliar o perfil demográfico dos pacientes submetidos a apendicectomia no Hospital Regional do Gama, no período de janeiro de 2016 a dezembro de 2017;

Classificar as complicações pós-operatórias das apendicectomias conforme Clavien-

Dindo;

Quantificar a necessidade de diagnóstico complementar de imagem;

Avaliar o perfil laboratorial do leucograma nos pacientes com apendicite aguda; Correlacionar as horas de evolução do quadro com os achados intra-operatórios.

\section{MATERIAIS E MÉTODOS}

\section{Materiais}

Registros cirúrgicos dos pacientes submetidos a cirurgia de emergência do HRG; Prontuário eletrônico da instituição, sistema TrakCare;

Foi mantido sigilo das informações dos pacientes no estudo, não sendo expostos na análise. 


\section{Métodos}

Foi realizado um estudo transversal retrospectivo dos pacientes submetidos a apendicectomia de urgência de janeiro de 2016 a dezembro de 2017 no serviço de urgência do Hospital Regional do Gama. Foram coletadas as seguintes variáveis: sexo, idade, horas de dor, presença de leucocitose e desvio a esquerda, utilização de ultrassonografia abdominal ou tomografia abdominal para diagnóstico, via de acesso cirúrgico, fases de evolução

intraoperatória, uso de antibiótico, uso de dreno abdominal, dias de internação, complicações pósoperatórias, classificação de Clavien-Dindo e necessidade e motivo de reabordagem cirúrgica. Foram excluídos deste estudo os pacientes com outras patologias diagnosticadas no intraoperatório e pacientes cujo prontuário apresentava-se sem descrição cirúrgica ou sem evolução intra-hospitalar.

No que se refere ao tempo de início dos sintomas, foi utilizado como parâmetro o tempo de evolução em horas de dor. 0 diagnóstico da doença foi obtido por meios de dados clínicos, laboratoriais e de imagem.

Quanto a classificação de Clavien-Dindo, foi categorizado de I a V. Sendo I correspondente a qualquer desvio do curso pós-operatório normal sem necessidade de intervenção para além da administração de antieméticos, antipiréticos, analgésicos, diuréticos, eletrólitos e fisioterapia. II complicações requerendo tratamento farmacológico com outros medicamentos além dos supracitados. III complicações requerendo intervenção cirúrgica, endoscópica ou radiológica. IV complicações com perigo de vida necessitando de internação em unidade de terapia intensiva. V óbito do paciente.

As análises estatísticas foram realizadas pelos programas Statistical Package for the Social SciencesSPSS versão 22 e Microsoftware excel 2016 para tabulação e organização de dados. Neste trabalho foi utilizado o nível de confiança usual de $5 \%(0,05)$, para análise dos testes estatísticos consideraremos p-valor < 0,05 como significativo, deste modo desconsideraremos a hipótese de igualdade $(\mathrm{Ho})$.

\section{RESULTADOS}

Entre os anos de 2016 e 2017 foram realizadas 374 cirurgias de apendicectomia pela emergência no Hospital Regional do Gama (HRG). Após análises estatísticas dos dados coletados inferimos os seguintes resultados. No ano de 2016 foram realizadas 184 cirurgias. No ano subsequente foi realizada 190, 14 cirurgias a mais, ou seja, um acréscimo de 3,2\% em relação ao ano anterior.

Conforme visto em literatura, a apendicite aguda foi mais prevalente em faixa etária menor ou igual a 30 anos, cerca de $58,8 \%$ dos casos descritos e nas faixas etárias maior ou igual a 70 anos apresentou um número ínfimo, em torno de $0,5 \%$ dos pacientes.

Essa enfermidade ainda é mais frequente em homens jovens se comparados a mulheres da mesma idade. Cerca de $68,7 \%$ dos casos analisados ocorreram em homens, o que também corrobora com as nossas referências.

Segundo avalição do perfil clínico por meio das informações da tabela 1 , vimos que grande parte do diagnóstico é feito nas primeiras 24 horas, cerca de $43,2 \%$ dos pacientes são submetidos ao atendimento médico nesse período. No entanto, os pacientes com mais de 72 horas de evolução também apresentaram um número relativo significativo considerável, por volta de $19,5 \%$ dos casos. E a presença da leucocitose foi algo bastante frequente nos pacientes analisados, cerca de $76,7 \%$, sendo que não necessariamente apresentaram desvio para a esquerda, apenas $7 \%$ dos pacientes possuíam leucocitose com desvio.

Já o uso de exames de imagem para diagnóstico teve pouca relevância para a investigação da doença. Do total de pacientes $61 \%$ não realizou ultrassonografia abdominal e $72,2 \%$ não foi submetido a tomografia de abdome para o diagnóstico da afecção.

A ressecção cirúrgica é o tratamento padrão outro para essa doença. Desta forma, observamos que a via cirúrgica mais realizada ainda é a cirurgia aberta com a laparotomia exploradora que possui incisão mediana que pode ou não ser infra ou transumbilical. Esta foi realizada em $54,8 \%$ das operações. Já a videolaparoscopia que é o método mais indicado devido aos benefícios propostos foi realizada em apenas $2,3 \%$ dos casos. Nas abordagens não complicadas ou com pouco tempo de evolução são utilizadas as incisões de Davis ou de McBrurney que correspondem respectivamente a $37,2 \%$ e $5,1 \%$ das incisões realizadas.

As fases de evolução da apendicite aguda são avaliadas durante o intraoperatório e são divididas em Não Complicada e Complicada, sendo que esta última ainda se subdivide em necrose, necrose com abscesso local, abscesso localizado e peritonite difusa. O número de apendicites não complicadas 
Tabela 1. Caracterização dos pacientes submetidos a apendicectomia de urgência no Hospital Regional do Gama (HRG). Brasília (DF) - 2016-2017. N:374.

\begin{tabular}{|c|c|c|c|}
\hline & $N(\%)$ & Média \pm DP & Mín - Máx \\
\hline \multicolumn{4}{|l|}{ Socio Demográfico } \\
\hline \multicolumn{4}{|l|}{ Ano } \\
\hline 2016 & $184(49,2)$ & & \\
\hline 2017 & $190(50,8)$ & & \\
\hline Fаiка Etária & & $30,13 \pm 12,97$ & $13-84$ \\
\hline$\leq 30$ anos & $220(58,8)$ & & \\
\hline $31|-| 50$ & $124(33,2)$ & & \\
\hline $51|-| 70$ anos & $28(7,5)$ & & \\
\hline$>70$ anos & $2(, 5)$ & & \\
\hline \multicolumn{4}{|l|}{ Sexо } \\
\hline Masculino & $257(68,7)$ & & \\
\hline Feminino & $117(31,3)$ & & \\
\hline \multicolumn{4}{|l|}{ Perfil Clinico } \\
\hline Evolução (Horas) & & $63,03 \pm 75,86$ & $1-720$ \\
\hline $0-24 h$ & $155(43,2)$ & & \\
\hline $25-48 h$ & $86(24,0)$ & & \\
\hline $49-72 h$ & $48(13,4)$ & & \\
\hline$>72 \mathrm{~h}$ & $70(19,5)$ & & \\
\hline \multicolumn{4}{|l|}{ Leucocitose 2 pontos } \\
\hline Sem leucocitose & $87(23,3)$ & & \\
\hline Com leucocitose & $287(76,7)$ & & \\
\hline \multicolumn{4}{|l|}{ Desvio à esquerda } \\
\hline Sem desvio a esquerda & $348(93,0)$ & & \\
\hline Com desvio a esquerda & $26(7,0)$ & & \\
\hline \multicolumn{4}{|l|}{ USG de Abdome total } \\
\hline Não realizou USG & $228(61,0)$ & & \\
\hline Realizou USG & $146(39,0)$ & & \\
\hline \multicolumn{4}{|l|}{ TC de abdome } \\
\hline Não realizou TC & $270(72,2)$ & & \\
\hline Realizou TC & $104(27,8)$ & & \\
\hline \multicolumn{4}{|l|}{ Via de acesso } \\
\hline Laparotomia exploradora & $193(54,8)$ & & \\
\hline Incisão de davis & $131(37,2)$ & & \\
\hline Incisão de Mcburney & $18(5,1)$ & & \\
\hline Videolaparoscopia & $8(2,3)$ & & \\
\hline Videolaparoscopia convertida em laparotomia exploradora & $1(, 3)$ & & \\
\hline Incisão de battle & $1(, 3)$ & & \\
\hline \multicolumn{4}{|l|}{ Fases de evolução } \\
\hline Abscesso localizado & $39(10,4)$ & & \\
\hline Não complicado & $193(51,6)$ & & \\
\hline Necrose & $41(10,7)$ & & \\
\hline Necrose e abscesso localizado & $45(12)$ & & \\
\hline Peritonite & $47(12,5)$ & & \\
\hline Sem dados & $10(2,6)$ & & \\
\hline
\end{tabular}


Continuação...

\begin{tabular}{|c|c|c|c|}
\hline \multicolumn{4}{|l|}{ Antibioticoterapia } \\
\hline Antibioticoterapia & $251(67,5)$ & & \\
\hline Sem uso de antibiotico & $112(30,1)$ & & \\
\hline Antibioticoprofilaxia & $9(2,4)$ & & \\
\hline Dias de Interação & & $4,35 \pm 5,61$ & $1-57$. \\
\hline 이-|1 dia & $74(20,3)$ & & \\
\hline $2 \mid-13$ dias & $158(43,3)$ & & \\
\hline $4 \mid-17$ dias & $86(23,6)$ & & \\
\hline $8|-| 15$ dias & $31(8,5)$ & & \\
\hline $16|-| 30$ dias & $13(3,6)$ & & \\
\hline$>30$ dias & $3(, 8)$ & & \\
\hline \multicolumn{4}{|l|}{ Complicação pos operatoria } \\
\hline \multicolumn{4}{|l|}{ Não } \\
\hline \multicolumn{4}{|l|}{ Sim } \\
\hline \multicolumn{4}{|c|}{ Classificação de Clavien Dindo } \\
\hline Grau I & $327(87,7)$ & & \\
\hline Grau II & $27(7,2)$ & & \\
\hline Grau III & $16(4,3)$ & & \\
\hline Grau V & $3(, 8)$ & & \\
\hline \multicolumn{4}{|l|}{ Necessidade de Dreno } \\
\hline Não & $309(83,1)$ & & \\
\hline Sim & $63(16,9)$ & & \\
\hline \multicolumn{4}{|l|}{ Rebordarem } \\
\hline Não & $351(93,9)$ & & \\
\hline Sim & $20(5,3)$ & & \\
\hline
\end{tabular}

Fonte: Autor

ainda é superior as outras fases chegando a 51,6\%. Embora, pacientes com peritonite e necrose com abscesso local foram, respectivamente, as complicações mais frequentes encontradas sendo $12,5 \%$ e $12 \%$.

O uso de antibióticos nas apendicites ainda é uma prática habitual. Dos 374 pacientes analisados 251 foram submetidos a antibioticoterapia, ou seja, em torno de $67,5 \%$ dos pacientes operados. Já a antibioticoprofilaxia é uma prática incomum nesse hospital, pois corresponde a $2,4 \%$ dos pacientes.

O período de internação é um fator que sugere bastante sobre a qualidade do procedimento cirúrgico e também acerca da gravidade das complicações. 0 tempo médio de internação ficou em torno de 4 dias, porém $43,3 \%$ dos pacientes operados ficaram um intervalo de 2 a 3 dias internados. Intervalos maiores como de 16 a 30 dias e maior que 30 dias indicam algum tipo de intercorrência durante o pós-cirúrgico justificando a extensão e isso representa 3,6 e $0,8 \%$, respectivamente.
Entre as complicações mais frequentes estão as infecções de ferida operatória e abscesso abdominal localizado que apresentou em 27 e 23 casos da amostra, respectivamente. A formação de bridas ou o desenvolvimento de sepse foram pouco frequentes nos pacientes operados nesse intervalo entre 2016 e 2017 chegando a 1 paciente em cada situação. Essas complicações ainda podem agravar levando a necessidade de reabordagem. 0 principal motivo para esta prática é também a formação de abscesso intracavitário com $63,16 \%$ da causa motivadora, seguida de evisceração e brida que corresponderam a exatos $31,58 \%$ e $5,26 \%$.

Durante o presente estudo, na tabela 1 , classificamos os pacientes conforme Clavien- Dindo, comparamos também $\mathrm{o}$ uso ou não de drenos abdominais e a necessidade de reabordagem. Os pacientes enquadrados pela classificação no grau I representa $87,7 \%$ da nossa amostra, o que significa que a maioria apresentou sintomas leves e de baixa complexidade no pós-operatório. Os demais parâmetros da classificação 
possuem valores relativos de baixa representatividade como é possível ver na tabela.

Ouso dedrenoesteve presenteemapenas $16,9 \%$ dos casos coletados. E o número de pacientes sem a necessidade de reabordagens foi de aproximadamente $93,9 \%$.

A partir da tabela 2 é possível dissociar a amostra total de 374 em um grupo para as complicações com 182 indivíduos e dele subdivide em outros dois: grupo $A$ (com dreno) e grupo $B$ (sem dreno). Sendo $p$-valor $<0,001$ para o teste de Kolmogorov-Smirnov.

O tempo de evolução para os pacientes do grupo A quando comparado aos do grupo $B$ foi maior, tendo em vista a correlação com o maior tempo de internação e esses dados juntos justificam a opção pela colocação do dreno. Comparando os dados obtidos do perfil sociodemográfico entre os grupos A e B podemos observar que nos anos de 2016 e 2017 o segundo grupo foi mais prevalente no desenvolvimento de apendicite aguda com complicação, tendo sido operado 34,8 e 32\% dos pacientes, consecutivamente a esses anos. A faixa etária, independente dos grupos envolvidos, manteve a característica de acometimento em público jovem. No entanto, o grupo $B$ apresenta uma incidência maior em todas as faixas etária em relação ao outro grupo.

Analisando o perfil clínico da tabela acima, vimos que os pacientes que pertence ao grupo $B$ apresentaram o tempo de evolução menor, cerca de $25 \%$ foi diagnosticado nas primeiras 24 horas de evolução e apenas 10,1\% tiveram diagnóstico tardio. Em contrapartida, o grupo $A$ apresentou somente $5,6 \%$ de diagnóstico precoce, mas $20,2 \%$ do grupo obteve diagnóstico após 72 horas de evolução. A presença da leucocitose foi mais prevalente no grupo B com cerca de $53 \%$ e em sua maioria sem desvio a esquerda.

Autilizaçãodosexamesdeimagemcomoaultrassonografia e a tomografia computadorizada foi pouco realizada no grupo $A$ em relação ao $B$ com respectivamente $13,3 \%$ e $26 \%$ de ecografias e $8,8 \%$ e $16,6 \%$ de tomografias. Já a via de acesso mais usada em ambos os grupos foi a laparotomia com $45 \%$ das abordagens no grupo B e $28,7 \%$ no grupo $A$. Entretanto, a videolaparoscopia não foi realizada no grupo $A$, o que corrobora para o surgimento de infecção do sitio, devido a cirurgia por vídeo ser uma medida protetiva ao seu aparecimento.

Nas fases de evolução, os pacientes do grupo B apresentou o estágio de evolução mais elevado em relação ao grupo $A$. Tendo $18 \%$ dos casos com peritonite e $22,5 \%$ com necrose apendicular. Além disso, as fases de evolução da apendicite foram mais prevalentes no grupo B. Logo, a antibioticoterapia e profilaxia também foi realizada neste mesmo grupo com $57,8 \%$ de uso de antibiótico nos pacientes operados e sem dreno.

Em contrapartida, o tempo de internação do grupo A foi relativamente maior se observamos com o grupo B. A maior parte dos pacientes sem a colocação do dreno ficaram no intervalo de 4 a 7 dias de internação o que corresponde a $27,1 \%$. Mas quando analisado os intervalos de tempo maiores vimos que o grupo com o uso de dreno possui maior prevalência. No intervalo de $16-30$ dias o grupo a chega a $5,6 \%$ dos pacientes, enquanto no grupo $B$ representa apenas $1,7 \%$.

Ainda na tabela 3 podemos ver que os dois grupos possuem maior prevalência no grau I da classificação sendo o grupo A $17,7 \%$ e o $B 61,3 \%$. Mas a medida que a gravidade vai aumentando conforme a classificação o grupo A demonstra uma frequência maior, embora no grau $V$ apenas o grupo B possuiu paciente que evoluiu ao óbito. Desta forma, percebe-se que o índice de reabordagem corrobora com os números achados na classificação de Clavien, já que o grupo A possui $7,8 \%$ das reabordagens.

Com a tabela 4 conseguimos inferir que a diferença entre o comportamento de cada grupo quando comparados as mesmas variáveis. Quando há um aumento na complexidade da enfermidade através do tempo de evolução, a necessidade de antibiótico, as complicações

Tabela 2. Teste de normalidade das variáveis quantitativas dos pacientes submetidos a apendicectomia de urgência no Hospital Regional do Gama (HRG). Brasília (DF) - 2016-2017. N:181

\begin{tabular}{llccc}
\hline \multirow{2}{*}{ Grupos } & & \multicolumn{3}{c}{ Kolmogorov-Smirnov } \\
\cline { 3 - 5 } & & Estatística & DF & P-valor \\
\hline \multirow{2}{*}{ Horas de evolução } & Grupo B (Sem Dreno) & 0,248 & 115 & $<0,001$ \\
& Grupo A (Com Dreno) & 0,221 & 59 & $<0,001$ \\
\multirow{2}{*}{ Dias de Internação } & Grupo B (Sem Dreno) & 0,308 & 115 & $<0,001$ \\
& Grupo A (Com Dreno) & 0,174 & 59 & $<0,001$ \\
\hline
\end{tabular}

Fonte: Autor 
Tabela 3. Caracterização dos dados Sociodemográficos e perfil Clínicos de pacientes submetidos a apendicectomia de urgência no Hospital Regional do Gama (HRG). Brasília (DF) - 2016-2017. N:181

\begin{tabular}{|c|c|c|c|c|}
\hline & \multicolumn{2}{|c|}{ Grupo B (Sem Dreno) } & \multicolumn{2}{|c|}{ Grupo A (Com Dreno) } \\
\hline & $N(\%)$ & Média \pm DP & $N(\%)$ & Média \pm DP \\
\hline \multicolumn{5}{|c|}{ Socio Demográficos } \\
\hline Ano & $27(14,9)$ & & $63(34,8)$ & \\
\hline 2016 & $33(18,2)$ & & $58(32,0)$ & \\
\hline 2017 & & $32,82 \pm 15,83$ & & $30,57 \pm 14,14$ \\
\hline \multicolumn{5}{|l|}{ Fаіка Etária } \\
\hline$\leq 30$ anos & $31(17,1)$ & & $69(38,1)$ & \\
\hline $31|-| 50$ & $21(11,6)$ & & $38(21,0)$ & \\
\hline $51|-| 70$ anos & $7(3,9)$ & & $13(7,2)$ & \\
\hline$>70$ anos & $1(, 6)$ & & $1(, 6)$ & \\
\hline \multicolumn{5}{|l|}{ Perfil Clinico } \\
\hline Evolução (Horas) & & $114,25 \pm 90,87$ & & $50,89 \pm 58,84$ \\
\hline $0-24 h$ & $10(5,6)$ & & $45(25,3)$ & \\
\hline $25-48 h$ & $4(2,2)$ & & $35(19,7)$ & \\
\hline $49-72 h$ & $9(5,1)$ & & $21(11,8)$ & \\
\hline$>72 \mathrm{~h}$ & $36(20,2)$ & & $18(10,1)$ & \\
\hline \multicolumn{5}{|l|}{ Leucocitose } \\
\hline Sem leucocitose & $10(5,5)$ & & $25(13,8)$ & \\
\hline Com leucocitose & $50(27,6)$ & & $96(53,0)$ & \\
\hline \multicolumn{5}{|l|}{ Desvio à esquerda } \\
\hline Sem desvio a esquerda & $55(30,4)$ & & $107(59,1)$ & \\
\hline Com desvio a esquerda & $5(2,8)$ & & $14(7,7)$ & \\
\hline \multicolumn{5}{|l|}{ USG de Abdome total } \\
\hline Não realizou USG & $36(19,9)$ & & $74(40,9)$ & \\
\hline RealizoU USG & $24(13,3)$ & & $47(26,0)$ & \\
\hline \multicolumn{5}{|l|}{ TC de abdome } \\
\hline Não realizou TC & $44(24,3)$ & & $91(50,3)$ & \\
\hline Realizou TC & $16(8,8)$ & & $30(16,6)$ & \\
\hline \multicolumn{5}{|l|}{ Via de acesso } \\
\hline Laparotomia exploradora & $49(28,7)$ & & $77(45,0)$ & \\
\hline Incisão de davis & $8(4,7)$ & & $28(16,4)$ & \\
\hline Incisão de mcburney & $1(6)$ & & $5(2,9)$ & \\
\hline Videolaparoscopia & $0(0,0)$ & & $3(1,8)$ & \\
\hline \multicolumn{5}{|l|}{ Fases de evolução } \\
\hline Abscesso localizado & $15(9,4)$ & & $24(15,0)$ & \\
\hline Necrose & $5(3,1)$ & & $36(22,5)$ & \\
\hline Necrose e abscesso localizado & $17(10,6)$ & & $16(10,0)$ & \\
\hline Peritonite & $18(11,3)$ & & $29(18,1)$ & \\
\hline \multicolumn{5}{|l|}{ Antibioticoterapia } \\
\hline Antibioticoterapia & $58(32,2)$ & & $104(57,8)$ & \\
\hline Sem uso de antibiotico & $2(1,1)$ & & $15(8,3)$ & \\
\hline Antibioticoprofilaxia & $0(, 0)$ & & $1(, 6)$ & \\
\hline
\end{tabular}




\begin{tabular}{|c|c|c|c|}
\hline Dias de Interação & & & $4,0 \pm 5,84$ \\
\hline 이-|1 dia & $0(0,0)$ & $16(9,0)$ & \\
\hline $2|-| 3$ dias & $1(6)$ & $46(26,0)$ & \\
\hline $4 \mid-17$ dias & $25(14,1)$ & $48(27,1)$ & \\
\hline $8|-| 15$ dias & $22(12,4)$ & $3(1,7)$ & \\
\hline $16|-| 30$ dias & $10(5,6)$ & $3(1,7)$ & \\
\hline$>30$ dias & $2(, 1)$ & $1(, 6)$ & \\
\hline \multicolumn{4}{|c|}{ Complicação pos operatoria } \\
\hline Não & $32(17,7)$ & $110(60,8)$ & \\
\hline Sim & $28(15,5)$ & $11(6,1)$ & \\
\hline \multicolumn{4}{|c|}{ Classificação de Clavien Dindo } \\
\hline Grau I & $32(17,7)$ & $111(61,3)$ & \\
\hline Grau II & $16(8,8)$ & $6(3,3)$ & \\
\hline Grau III & $12(6,6)$ & $2(1,1)$ & \\
\hline Grau IV & $0(0,0)$ & $0(0,0)$ & \\
\hline Grau V & $0(0,0)$ & $2(1,1)$ & \\
\hline \multicolumn{4}{|l|}{ Reabordagem } \\
\hline Não & $46(25,6)$ & $116(64,4)$ & \\
\hline Sim & $14(7,8)$ & $4(2,2)$ & \\
\hline
\end{tabular}

Fonte: Autor

pós-cirúrgicas e o tempo de internação o grupo com o uso de dreno segue a mesma tendência e aumentam sua prevalência.

Durante a investigação foi possível desmembrar as variáveis da complicação cirúrgica pós-operatória para avaliá-las. A tabela acima sugere que o grupo A esteve mais susceptível a formação dos abscessos e infecções do sítio cirúrgico. Além disso, apresentou um maior índice de reabordagem, cerca de $7,7 \%$ dos pacientes desse grupo. Porém, a evolução ao óbito foi uma característica única do grupo $\mathrm{B}$. Os dados citados ainda podem ser demonstrados conforme os gráficos 3 e 4 a baixo.

Com já citado acima, o tempo de evolução e os dias de internação são indicadores bastante relevantes para entender a gravidade e a complexidade da apendicectomia e das suas complicações subsequentes. Na tabela 5, sendo $p<0,001$, observa-se, que para o grupo com dreno, a média de tempo de evolução foi de 114 horas e a duração das internações foi de aproximadamente de 11 dias. Como já esperado, o grupo $B$, sem o dreno, o tempo médio de evolução da doença e de internação foram menores, 56,86 horas e cerca de 4 dias, respectivamente.

Quvanto maior o tempo de evolução, maior será a probabilidade de o paciente evoluir com algum tipo de complicação nas fases de evolução. Conforme demonstrado na tabela 6 , a medida que o intervalo da evolução da doença aumenta de modo geral o percentual de comprometimento a cada fase. Na tabela seguinte, tabela 7, é possível estabelecer um parelelo entre as mesmas variáveis da tabela anterior. No entanto, quando comparo as horas de evolução, com p-valor $=0,979$, há uma correlação muito forte entre as variáveis e quando comparo as horas de evolução aos dias de internação observa-se uma correlação moderada.

A classificação de Clavien-Dindo, já mencionada também foi analisada comparando aos dois grupos, $A$ e $B$, para $p<0,001$. 0 grupo $A$ apresenta pouca prevalência ao grau I se correlacionarmos com o grupo $B$, no entanto, a medida que vai aumentando a complexidade da classificação o grupo A volta a apresentar valores relativos maiores, principalmente nos graus II e III como é possível observar na tabela 8.

\section{DISCUSSÃO}

A apendicite aguda é uma emergência cirúrgica muito frequente nas unidades de pronto atendimento em todo o mundo. Ela pode apresentar-se de variadas formas, mas com sintomas inespecíficos que com a progressão da doença evolui para sinais característicos. Com base nas referências usadas e mediante aos resultados apresentados, nota-se que a faixa etária para 
Tabela 4. Análise de associação (homogeneidade) entre os grupos de estudo e as variáveis clinicas dos pacientes submetidos a apendicectomia de urgência no Hospital Regional do Gama (HRG). Brasília (DF) - 2016-2017. N:181

\begin{tabular}{|c|c|c|c|}
\hline & Grupo A (Com Dreno) & Grupo B (Sem Dreno) & P-valor \\
\hline & N (\%) & $N(\%)$ & \\
\hline \multicolumn{4}{|c|}{ Perfil Clinico } \\
\hline Dias de Interação & & & $<0,001$ \\
\hline 이-|1 dia & $0(0,0)$ & $16(9,0)$ & \\
\hline $2 \mid-13$ dias & $1(0,6)$ & $46(26,0)$ & \\
\hline $4 \mid-17$ dias & $25(14,1)$ & $48(27,1)$ & \\
\hline $8|-| 15$ dias & $22(12,1)$ & $3(1,7)$ & \\
\hline $16|-| 30$ dias & $10(5,5)$ & $3(1,7)$ & \\
\hline$>30$ dias & $2(1,1)$ & $1(, 6)$ & \\
\hline Evolução (Horas) & & & $<0,001$ \\
\hline $0-24 h$ & $10(5,6)$ & $45(25,3)$ & \\
\hline $25-48 h$ & $4(2,2)$ & $35(19,7)$ & \\
\hline $49-72 h$ & $9(5,1)$ & $21(11,8)$ & \\
\hline$>72 \mathrm{~h}$ & $36(20,2)$ & $18(10,1)$ & \\
\hline Antibioticoterapia & & & 0,105 \\
\hline Antibioticoterapia & $58(32,3)$ & $104(57,8)$ & \\
\hline Sem uso de antibiotico & $2(1,1)$ & $15(8,3)$ & \\
\hline Antibioticoprofilaxia & $0(0,0)$ & $1(, 6)$ & \\
\hline Complicação pos operatoria & & & $<0,001$ \\
\hline Sim & $28(15,1)$ & $11(6,1)$ & \\
\hline Não & $32(17,1)$ & $110(60,8)$ & \\
\hline \multicolumn{4}{|c|}{ Complicação pos operatoria } \\
\hline Abscesso abdominal & & & $<0,001$ \\
\hline Sim & $17(9,9)$ & $3(1,7)$ & \\
\hline Não & $43(23,2)$ & $118(65,2)$ & \\
\hline Infecçao de ferida operatoria & & & $<0,001$ \\
\hline Sim & $14(7,7)$ & $6(3,3)$ & \\
\hline Não & $46(25,2)$ & $115(63,5)$ & \\
\hline Evisceraçao & & & 0,154 \\
\hline Sim & $1(0,6)$ & $0(0,0)$ & \\
\hline Não & $59(32,3)$ & $121(66,9)$ & \\
\hline Óbito & & & 0,317 \\
\hline Sim & $0(0,0)$ & $2(1,1)$ & \\
\hline Não & $60(33,3)$ & $119(65,7)$ & \\
\hline Hemorragia & & & 0,611 \\
\hline Sim & $1(6,6)$ & $1(, 6)$ & \\
\hline Não & $59(32,3)$ & $120(66,3)$ & \\
\hline Evisceraçao & & & 0,072 \\
\hline Sim & $3(1,1)$ & $1(, 6)$ & \\
\hline Não & $57(31,3)$ & $120(66,3)$ & \\
\hline Sepse & & & 0,48 \\
\hline Sim & $0(0,0)$ & $1(, 6)$ & \\
\hline Não & $60(33,3)$ & $120(66,3)$ & \\
\hline Brida & & & 0,48 \\
\hline Sim & $0(0,0)$ & $1(, 6)$ & \\
\hline Não & $60(33,3)$ & $120(66,3)$ & \\
\hline Rebordarem & & & $<0,001$ \\
\hline Sim & $14(7,7)$ & $4(2,2)$ & \\
\hline Não & $46(25,2)$ & $116(64,4)$ & \\
\hline
\end{tabular}

Fonte: Autor 
Tabela 5. Análise de comparação entre os grupos de estudo e o tempo de evolução (Horas) e Dias de Internação dos pacientes submetidos a apendicectomia de urgência no Hospital Regional do Gama (HRG). Brasília (DF) - 2016-2017. N:181.

\begin{tabular}{lccc}
\hline & Grupo A (Com Dreno) & Grupo B (Sem Dreno) & \multirow{2}{*}{ P-valor } \\
\cline { 2 - 3 } & Média \pm DP & Média \pm DP & \\
\hline Horas de evolução & $56,89 \pm 58,84$ & $114,25 \pm 90,87$ & $<0,001$ \\
Dias de Internação & $4,30 \pm 5,84$ & $10,85 \pm 7,77$ & $<0,001$ \\
\hline
\end{tabular}

Fonte: Autor

Tabela 6. Análise de associação entre a evolução (horas e as fases de evolução dos pacientes) submetidos a apendicectomia de urgência no Hospital Regional do Gama (HRG). Brasília (DF) - 2016-2017. N:181.

\begin{tabular}{|c|c|c|c|c|c|}
\hline & \multicolumn{4}{|c|}{ Evolução (Horas) } & \multirow{3}{*}{ P-valor } \\
\hline & $0-24 h$ & $25-48 h$ & $49-72 h$ & $>72 h$ & \\
\hline & $N(\%)$ & $N(\%)$ & $N(\%)$ & $N(\%)$ & \\
\hline Fases de evolução & & & & & $<0,001$ \\
\hline Abscesso localizado & $14(8,9)$ & $3(1,9)$ & $5(3,2)$ & $15(9,6)$ & \\
\hline Necrose & $22(14,0)$ & $9(5,7)$ & $7(4,5)$ & $3(1,9)$ & \\
\hline Necrose e abscesso localizado & $7(4,5)$ & $9(5,7)$ & $3(1,9)$ & $14(8,9)$ & \\
\hline Peritonite & $7(4,5)$ & $10(6,4)$ & $13(8,3)$ & $16(10,2)$ & \\
\hline
\end{tabular}

Fonte: Autor

Tabela 7. Análise de correlação entre a evolução horas e as fases de evolução dos pacientes submetidos a apendicectomia de urgência no Hospital Regional do Gama (HRG). Brasília (DF) - 2016-2017. N:181.

\begin{tabular}{lcccc}
\hline & & Idade & Horas de evolução & Dias de Internação \\
\hline \multirow{2}{*}{ Idade } & CC & 1,000 & $-0,002$ & 0,05 \\
& P-valor & & 0,979 & 0,513 \\
Horas de evolução & CC & & 1,000 & $0,381 \% *$ \\
Dias de Internação & P-valor & & & 0,000 \\
\hline
\end{tabular}

Fonte: Autor

Tabela 8. Análise de associação entre os grupos e a classificação de Clavien-Dido pacientes submetidos a apendicectomia de urgência no Hospital Regional do Gama (HRG). Brasília (DF) - 2016-2017. N:181.

\begin{tabular}{lccc}
\hline & Grupo A (Com Dreno) & Grupo B (Sem Dreno) & P-valor \\
\hline Classificação de Clavien Dindo & $\mathbf{N}(\%)$ & $\mathbf{N}(\%)$ & $<0,001$ \\
\hline Grau I & $32(17,7)$ & $111(61,3)$ & \\
Grau II & $16(8,8)$ & $6(3,3)$ & \\
Grau III & $12(6,6)$ & $2(1,1)$ & \\
Grau V & $0(0,0)$ & $2(1,1)$ & \\
\hline
\end{tabular}

Fonte: Autor

acometimento é de adolescentes e adultos jovens e mais raramente em idosos. Além disso, é ser um evento mais frequente em pacientes do sexo masculino. $1,2,11-13$

A complexidade dessa doença está ligada ao tempo de progressão até o momento do diagnóstico e consequentemente da intervenção. 0 diagnóstico feito precocemente corrobora para diminuir as taxas de morbimortalidade ligada a evolução da doença. ${ }^{8}$
A evolução da apendicite, hoje, é dividida em "Não complicada" e "Complicada", sendo que esta última ainda se subdivide em outras quatro modalidades: necrose apendicular, necrose com abscesso localizado, abscesso e peritonite difusa. ${ }^{11-13}$

De acordo com os dados analisados e bibliografia, a população idosa apesar de ser a menos prevalente é quem mais sofre as complicações mais graves 
decorrentes da apendicite. Isso pode estar relacionado ao número de comorbidades adquiridas ao longo da vida e ao tempo que se leva para fazer o diagnóstico que, segundo Almeida et al., a complicação mais frequente é a perfuração do ceco. ${ }^{1,11-13,}$

Como já citado, o diagnóstico precoce está intimamente ligado as complicações advindas da enfermidade. 0 reconhecimento do quadro é eminentemente clínico, mas também pode contar com o auxílio de exames laboratoriaise de imagem. Asintomatologia característica da apendicite aguda se dar pela dor periumbilical que migra para a fossa ilíaca direita, anorexia e náuseas e vômitos. Ademais, há sinais semiológicos que podem ajudar na identificação da doença como o sinal de Blumberg, que é a dor a descompressão, e Lenander, diferença na variação da temperatura do ânus em relação a boca. ${ }^{8}$

Já os achados no exame laboratorial é a leucocitose com ou sem o desvio a esquerda, além da analise urinaria que possibilita ver acetona como resultado da anorexia e também a leucocitose urinaria. Os exames de imagens usados para auxiliar o diagnóstico são a ultrassonografia e a tomografia computadorizada. $\mathrm{O}$ ultrassom ainda que amplamente utilizado existe controvérsias a despeito da técnica de diagnóstico. Ele possui aproximadamente entre $40 \%$ e $100 \%$ de sensibilidade e a sua especificidade é em torno de $47 \%$ a $99 \%$. No entanto, é um método operador-dependente. ${ }^{8}$

A tomografia é uma boa opção de exame para avaliar o paciente com quadro clínico inespecífico. Porém, o seu uso é questionável devido a exposição à radiação que pode ser nociva aos pacientes pelo excesso de exposições. 0 escore de Alvarado pode ajudar na interpretação da tomografia quando somados 7 ou mais critérios. ${ }^{8}$

O tratamento da apendicite aguda já é bem estabelecido. A intervenção cirúrgica, apendicectomia, é o método mais eficaz para o tratamento dessa doença. A videolaparoscopia é a via cirúrgica mais realizada no mundo desde de 1983 e se estabeleceu como padrão ouro devido aos benefícios implicados a técnica. 0 seu uso interfere diretamente na redução do número de infecções pós-cirúrgicas e da dor, diminuição das complicações, recuperação mais rápida e, ainda, bons resultados estéticos. ${ }^{9,14,18}$

O tempo de internação do paciente submetido a apendicectomia é bastante variável e depende de fatores como tempo de evolução da doença, via de intervenção cirúrgica, complicações pós-operatórias, uso ou não de dreno abdominal e até mesmo da idade do paciente. As análises feitas neste presente estudo, em uma população de 374 pacientes, a média da duração das internações foi de 4 dias, porém em torno de $43,3 \%$ ficou de 2 a 3 dias internado. ${ }^{11,12,13}$

Este estudo demonstrou também que nos pacientes complicados o tempo de internação foi de intervalos maiores que 7 dias e ainda quando comparados aos pacientes com dreno a internação se estendeu mais. ${ }^{12}$

Desta forma, os pacientes complicados foram divididos pela utilização ou não dos drenos abdominais em 2 grupos. Foi possível observar que, embora, ambos os grupos sejam frutos de complicações, os pacientes em usos de drenos abdominais estiveram mais susceptíveis a outras complicações no pós-operatório. A amostra de indivíduos com dreno é menor quando comparada aos sem dreno, mas, ainda assim, foi possível inferir alguns dados.

Mediante a comparação dos grupos $A$ e $B$, respectivamente com e sem dreno, observa- se que, mesmo os pacientes idosos estarem mais relacionados a presença de complicações, o uso de dreno foi mais prevalente nos indivíduos jovens. Ao avaliar as fases de evolução da doença nesses grupos nota-se que comportam de forma diferente. Nos pacientes com dreno o índice de complicação é relativamente maior que nos pacientes sem a necessidade de drenagem, isso entra em acordo com a literatura investigada.

Segundo Wada ${ }^{14}$, Li etal. ${ }^{17}$ eHiramatsu et al. ${ }^{18}$, a quantidade de abscessos e a necessidade de drenagem foi menor nos grupos sem o dreno intracavitário. Puderam observar ainda que até em apendicites graves o uso do dreno não é totalmente recomendado. Li et al. ${ }^{17}$,

ainda sugere que os resultados insatisfatórios da drenagem estejam ligados a fatores como obstrução do dreno por coágulos ou tampões de fibrina, fluxo de vazão menor que a produção de secreção e também devido ao tamanho da amostra. ${ }^{14,15,17,18}$

Para a utilização do dreno abdominal é preciso avaliar alguns critérios no intuito de indicá-lo no momento mais oportuno o possível. A sua utilização está recomendada nos casos de abscesso bem localizados e delimitados, na presença de fístulas ou secreções residuais e formação de hematomas. Entretanto, a drenagem abdominal está contraindicada quando houver sinais de peritonite, reação alérgica sabidamente ao material e na incisão cirúrgica. Além disso, o cirurgião deve se propor materiais flexíveis, suaves e menos irritantes. Quando introduzido 
deve-se ter o cuidado de posicionar a fim de que não danifique nenhuma estrutura adjacente e após lavagem exaustiva da cavidade. . $^{15,17,19}$

A classificação de Clavien-Dindo foi uma ferramenta desenvolvida para auxiliar graduação e avaliação da complexidade das complicações advindas da apendicite aguda. Para Moreira et al. ${ }^{2}$, ela possui um método fácil, acessível e aplicável em qualquer região do mundo. Isso devido basear-se no tratamento e nas complicações póscirúrgicas do paciente. ${ }^{16}$

As fases de evolução juntos a classificação possuem alto valor preditivo para qualificar a complexidade da doença. Com isso também pode-se avaliar a terapia adequada e o prognóstico dos indivíduos acometidos pela enfermidade. Se analisarmos os grupos A e B mais uma vez, no entanto, sob a ótica classificação comparada ao tempo de evolução e internação, percebe-se que o grupo com o dreno possuiu maior classificação em gravidade e um tempo de evolução maior, consequentemente ficou mais tempo internado em relação ao grupo sem o dreno. ${ }^{2,16}$

\section{CONCLUSÃO}

No presente estudo foi observado que a maioria da população acometida pela patologia são de adultos jovens do sexo masculino. Indivíduos com idade mais avançada tem maior tempo de evolução clínica e consequentemente maiores taxas de apendicite complicada.

Além disso, pode-se inferir que o uso do dreno abdominal na apendicite não apresentou muitos benefícios. 0 seu uso acarretou ao paciente maior taxa de complicações pós-operatórias que outrora poderiam ser minimizados ou até mesmo evitado, como infecção de ferida operatória e abscesso abdominal localizado. Ficou evidente que o tempo de internação e, consequentemente, recuperação dos pacientes que fizeram o uso de dreno foi maior. Após a análise estatística dos dados aferidos comparando a população com ou sem dreno, encontramos uma evidência de $p<0,001$, ou seja, a probabilidade de eventos similares aos estudados acontecer e reproduzir o mesmo efeito é extremamente alta. Desta forma, a utilização de dreno no pós-cirúrgico da apendicectomia possui baixo índice de evidência para indicá-lo como uma manobra segura.

As implicações das complicações desenvolvidas também interferem diretamente nos hospitais onde são realizados os processos, uma vez que onera a instituição com o aumento dos custos de internação e da terapia utilizada.

De fato, a apendicectomia videolaparoscópica é o melhor tratamento que pode ser oferecido ao paciente nessa condição. No entanto, nota-se que não é uma prática muito realizada no hospital deste estudo, o que conflui para o desenvolvimento das complicações pós-cirúrgicas que dentre elas a infecção do sítio cirúrgico é a mais comum.

\section{REFERÊNCIAS BIBLIOGRÁFICAS}

1. IAMARINO, Ana Paula; JULIANO, Yara. Fatores de risco associados à complicações de apendicite aguda. Revista Colégio Brasileiro Cirurgia; 44(6), 560- 566, 2017. https://www.scielo.br/pdf/rcbc/v44n6/pt_1809-4546rcbc-44-06-00560.pdf

2. MOREIRA, Luis; GARBIN, Henrique. Fatores preditores de complicações pós-operatórias em apendicectomias. Revista Colégio Brasileiro Cirurgia; 45 (5),1920, 2018. https://www.scielo.br/scielo.php?script=sci_ arttext\&pid=S0100-69912018000500155

3. BORGES, Paulo Sérgio Gomes Nogueira; LIMA, Marilia de Carvalho; NETA, Gilliatt Hanois Falbo. Validação do Escore de Alvarado no diagnóstico de Apendicite Aguda em Crianças e Adolescentes no Instituto Materno Infantil de Pernambuco, IMIP. Revista Brasileira Saúde Materna Infantil, Recife, 3(4):439- 445,2003. https://www. scielo.br/scielo.php?script=sci_abstract\&pid=S151938292003000400008\&lng=en\&nrm=iso\&tlng=pt

4. RODRIGUES, Célio Fernando de Sousa; ROCHA, Amaury Clemente da; Rodrigues, Amanda Karine Barros et al. Correlação entre a Escala de Alvarado e o aspecto macroscópico do apêndice em pacientes com apendicite. Revista do Colégio Brasileiro de Cirurgiões 41(5); 336340; Maceió-AL, Brasil;2014. https://www.scielo.br/pdf/ rcbc/v41n5/pt_0100-6991-rcbc-41-05-00336.pdf

5. Addiss DG, Shaffer N, Fowler BS, Tauxe RV. The epidemiology of appendicitis and appendectomy in the United States. Am J Epidemiol.132(5):910-25;1990. https://pubmed.ncbi.nlm.nih.gov/2239906/

6. MonteiroMSS, ReinaldoGC. Prevalência de apendicectomias e análise dos histopatológicos em hospital de urgências de Teresina. J. Ciênc. Saúde [internet]. 2018 [acesso em: 10 de Outubro de 2018];1(1):86-95. https://revistas.ufpi.br/ index.php/rehu/article/view/6762

7. SABISTON JR., D. C., TOWNSEND, M. C. Tratado de Cirurgia, A Base Biológica da Prática Cirúrgica Moderna. 19. a ed. Rio de Janeiro-RJ; Editora Elsevier, 2015.

8. ALVARADO, Alfredo. How to improve the clinical diagnosis of acute appendicitis in resource limited settings. Alvarado World Journal of Emergency Surgery, 11:16; 2016. https:// pubmed.ncbi.nlm.nih.gov/27118990/

9. GOMES, Carlos Augusto. CLASSIFICAÇÃO E TRATAMENTO LAPAROSCÓPICO DA APENDICITE AGUDA. Faculdade de Medicina -UFMG. Belo Horizonte-BH, Brasil;2009. https:// www.scielo.br/scielo.php?script=sci_arttext\&pid=S010069912006000500006 
10. BORGES, Paulo Sérgio Gomes Nogueira; LIMA, Marilia de Carvalho; NETA, Gilliatt Hanois Falbo. Validação do Escore de Alvarado no diagnóstico de Apendicite Aguda em Crianças e Adolescentes no Instituto Materno Infantil de Pernambuco, IMIP. Revista Brasileira Saúde Materna Infantil, Recife, 3(4):439-445,2003. https://www. scielo.br/scielo.php?script=sci_abstract\&pid=S1519$38292003000400008 \&$ lng=en\&nrm=iso\&tlng=pt

11. LIMA, Amanda Preira; VIEIRA, Felipe José; OLIVEIRA, Gabriela Procópio de Moraes, et al. Perfil clínicoepidemiológico da apendicite aguda: análise retrospectiva de 638 casos. Revista do Colégio Brasileiro de Cirurgiões. Juiz de Fora-MG, Brasil, 43(4);248-253;2016. https:// www.scielo.br/scielo.php?script=sci_arttext\&pid=S010069912016000400248\&lng=pt\&nrm=iso\&tlng=pt

12. ALMEIDA, Marcelo Wilson Rocha; JOÃO, Angela Trevisan; OLIVEIRA, Fernanda Silveira; et al. Influência da idade no tempo de internação e no grau evolutivo das apendicites agudas. Revista do Colégio Brasileiro de Cirurgiões, Vol. 33 - n5, Set./Out, 2006. [acesso em: 05 de Setembro de 2018]. https://www.scielo.br/scielo.php?pid=S0100$69912006000500007 \&$ script=sci_abstract\&tlng=pt

13. FISHER, Carlos. Apendicite aguda: existe relação entre o grau evolutivo, idade e tempo de internação? Revista do Colégio Brasileiro de Cirurgiões: Vol. 32 - No 3, Mai. I Jun. 2005. https://www.scielo.br/scielo.php?pid=S0100$69912005000300007 \&$ script=sci_abstract\&tlng=pt

14. WADA, Tomoko. Abdominal drainage may be a risk factor for surgical site infection following appendectomy [letter]. Asian Journal of Surgery (2019) 42, 897 e 898. https:// pubmed.ncbi.nlm.nih.gov/31235202/
15. LIMA, Sabrina; SARTORI, Priscila Ely; SOUZA, Hamilton Petry. DRENOS ABDOMINAIS: INDICAÇÕES E UTILIZAÇÃO NA PRÁTICA CIRÚRGICA. Acta méd.(Porto Alegre), 2013. https://pesquisa.bvsalud.org/portal/resource/pt/ biblio-880496

16. MOREIRA, Luis Fernando; PESSÔA, Marcelo Castro Marçal; MATTANA, Diego Sachet; SCHMITZ, Fernando Fernandes; VOLKWEIS, Bernardo Silveira; ANTONIAZZI, Jorge Luiz; RIBEIRO, Liacyr. Adaptação cultural e teste da escala de complicações cirúrgicas de Clavien-Dindo traduzida para o Português do Brasil. Rev. Col. Bras. Cir. vol.43 no.3 Rio de Janeiro May./June 2016. https://www.scielo.br/scielo. php?pid=S0100 $\quad$ 69912016000300141\&script=sci_ arttext\&tlng=pt

17. LI, Z; ZHAO, L; CHENG, Y; CHENG, N; DENG, Y. Abdominal drainage to prevent intra-peritoneal abscess after open appendectomy for complicated appendicitis. Cochrane Database of Systematic Reviews 2018, Issue 5. Art. No.: CD010168. https://pubmed.ncbi.nlm.nih.gov/29741752/

18. HIRAMATSU, Kosuke; TODA, Shigeo; TATE, Tomohiro; FUKUI, Yudai; TOMIZAWA, Kenji; HANAOKA, Yutaka; MORIYAMA, Jin; MATOBA, Shuichiro; KUROYANAGI, Hiroya. Can laparoscopic appendectomy be safely performed by surgical residents without prior experience of open appendectomy? Asian J Surg. 2018;41: 270 e 273. https:// pubmed.ncbi.nlm.nih.gov/28139339/

19. D'ACAMPORA, AJ; ELY, JB; RUSSI, RF; OLIVEIRA, SCV; SGROTT, SM. Dreno na cavidade abdominal: uso ou não uso? Rev. Med. Res., Curitiba, v.15, n.2, p.115-119,abr./jun. 2013. http://crmpr.org.br/publicacoes/cientificas/index. php/revista-do- medico-residente/article/view/401 